\section{Avaliação da conduta de profilaxia antirrábica indicada para pessoas envolvidas em agravos com cães e gatos no município de Jaboticabal, SP, no período de 2000 a 2006}

\section{Evaluation of rabies post-exposure prophylaxis in humans injured by dogs and cats in the municipality of Jaboticabal, SP, from 2000 through 2006}

\section{Danila Fernanda Rodrigues Frias \\ Sonia Luisa Silva Lages}

\section{Adolorata Aparecida Bianco Carvalho}

Departamento de Medicina Veterinária Preventiva e Reprodução Animal da Faculdade de Medicina Veterinária da Universidade Estadual Paulista "Julio de Mesquita Filho". Jaboticabal, SP.

Trabalho realizado na Universidade Estadual Paulista (UNESP), Campus de Jaboticabal/SP, em parceria com a Secretaria Municipal de Saúde do Município.

Conflito de interesse: nada a declarar.

Financiamento: Bolsa de doutorado - CAPES

Correspondência: Danila Fernanda Rodrigues Frias. Medicina Veterinária Preventiva. Via de Acesso Prof. Paulo Donatto Castelane, S/N, Rural, Jaboticabal, SP CEP 14.884-900. E-mail: danilafrias@yahoo.com.br

\section{Resumo}

Objetivando analisar as indicações de profilaxia antirrábica humana no Município de Jaboticabal-SP, foi realizado um estudo retrospectivo descritivo no período de 2000 a 2006, com levantamento de dados registrados nas fichas de investigação de atendimento e cálculo do custo com as vacinas destinadas à profilaxia pós-exposição. Constatou-se que 2.493 pessoas agredidas por animais foram submetidas à profilaxia com uso de vacina, num total de 7.108 doses e um custo de $\mathrm{R} \$ 179.105,14$. Da totalidade de casos notificados, 2.184 (71,5 \%) foram causados por cães e gatos clinicamente sadios no momento da agressão e que assim se mantiveram durante o período de observação, a qual foi feita pela própria vítima ou pelo dono do animal. Considerando este fato e também a situação epidemiológica da raiva no Município, pode-se inferir que essas vítimas poderiam ter sido dispensadas da profilaxia; entretanto, apenas 464 o foram, ou seja, 1.720 pessoas podem ter recebido vacina sem necessidade, ou seja 4.590 doses a um custo de $\mathrm{R} \$ 114.420,81$. Em comparação com os números de outros municípios do Estado de São Paulo e com a média nacional, constata-se que o número de profilaxias pós-exposição contra raiva é muito alto em Jaboticabal, evidenciando que na conduta não se considerou o estado do agressor e a condição do Município de área controlada para raiva. Recomenda-se conscientização e capacitação permanentes das equipes de saúde pública quanto à epidemiologia da doença e à necessidade de observação adequada do animal agressor. É essencial a integração dos serviços médicos e veterinários no atendimento às vitimas, visando uma melhor avaliação do caso para que a decisão de se instituir ou não a profilaxia pós-exposição seja feita com critério e segurança.

Palavras-chave: Raiva. Profilaxia pós-exposição. Vacina contra raiva. 


\section{Abstract}

The present study aimed to evaluate rabies post-exposure prophylaxis (PEP) in humans in the municipality of Jaboticabal, São Paulo from 2000 through 2006. A descriptive and retrospective study was conducted by collecting data available in patient records. Vaccination costs were also calculated; 2,493 patients injured by animals received PEP, totaling $\mathrm{R} \$ 179,105.14$ and 7,108 doses; $2,184(71.5 \%)$ out of the total reports were caused by dogs and cats clinically healthy at the moment of the attack. These animals remained sound throughout the 10-day observation period. The observation was conducted by the victim or by the owner. Considering animal observation and the epidemiological status of rabies in the municipality, all of these patients could have been dismissed from PEP treatment. Instead, only 464 were dismissed, meaning that 1,720 patients were unnecessarily vaccinated. An estimated 4,590 doses and $\mathrm{R} \$ 114,420.81$ could have been saved. In comparison with rates of other municipalities of the State of São Paulo and with the national mean, the number of PEP in Jaboticabal is very high and it became evident that management evaluated neither the health status of the animal nor the epidemiological status of the area for rabies. Permanent awareness and education of public health professionals with respect to rabies epidemiology and the need to perform correct observation of the aggressors are recommended. It is essential that medical and veterinary services be integrated to provide better assessment of cases and safer decisions on the institution of PEP.

Keywords: Rabies. Post-exposure prophylaxis. Rabies vaccine.

\section{Introdução}

Embora seja uma doença perfeitamente passível de prevenção, a raiva continua sendo uma importante causa de mortalidade humana em muitas partes do mundo, representando, além disso, um grande desafio para as autoridades sanitárias ${ }^{1-3}$.

A importância que a raiva assume para a saúde pública reside no fato de se tratar de uma antropozoonose invariavelmente letal, e no impacto psíquico e emocional que causa nas pessoas mordidas, mediante o temor de contrair a doença ${ }^{4,5}$.

A raiva apresenta três ciclos: o urbano, onde a enfermidade se mantém em algumas áreas em cães e gatos; o rural, representado por animais de produção, especialmente os herbívoros (bovinos, equídeos, caprinos e ovinos), onde o morcego hematófago é o principal transmissor; e o silvestre, representado principalmente pelos carnívoros, como raposas e guaxinins, além de primatas não humanos (saguis) e morcegos?

O ciclo urbano da doença continua sendo o mais importante para a manutenção da raiva humana no mundo, especialmente nos países em desenvolvimento. Em áreas onde as medidas de controle não atingem seu objetivo, a espécie de maior relevância epidemiológica para a transmissão do vírus é a canina, principal reservatório e fonte de infecção, seguida pelos quirópteros ${ }^{4,8-10}$. No Brasil, em 2006, houve uma mudança no perfil epidemiológico da raiva, tendo o morcego hematófago assumido o papel de principal transmissor da doença a humanos; surtos no Maranhão e no Pará ocorreram devido a ataques de Desmodus rotundus a moradores de comunidades no interior desses Estados. Com a solução dos casos, novamente o cão passou a ser o principal transmissor. Ressalta-se que os morcegos não hematófagos também são importantes na cadeia epidemiológica, podendo participar na transmissão da doença aos humanos ${ }^{11}$.

O número de pessoas acometidas pela enfermidade ainda é elevado no mundo. Estima-se 55 mil mortes por ano ${ }^{1,3}$, princi- 
palmente em países menos desenvolvidos, como as áreas rurais da Ásia e da África. Na Ásia morrem cerca de 31 mil pessoas por ano vítimas da raiva e são gastos, anualmente, 560 milhões de dólares com profilaxia pós-exposição ${ }^{6}$. Na América Latina, até o ano 2000, o número de mortos aproximavase de 100 por ano e a média anual de profilaxia antirrábica pós-exposição era de $500 \mathrm{mil}$ pessoas $^{12}$.

Devido ao fato de a raiva ser fatal, a profilaxia pós-exposição é extremamente importante ${ }^{13}$. Nos países menos desenvolvidos, onde a maior parte dos casos de raiva humana acontece devido à raiva canina, as mortes ocorrem principalmente pela falta de acesso a agentes biológicos adequados e necessários para um tratamento profilático conveniente. Em países desenvolvidos, embora a raiva em animais domésticos já esteja controlada, a doença continua sendo um risco para a população, devido ao fato de animais silvestres (canídeos, felídeos e morcegos) estarem migrando para áreas urbanas; as vacinas são acessíveis e as poucas mortes humanas ocorrem devido à ignorância ou ao não reconhecimento da exposição, sendo de grande importância a conscientização e educação, tanto do público quanto dos profissionais da área de saúde ${ }^{14,15}$.

A instituição de profilaxia pós-exposição deve ser adequada, após a análise do caso, mediante anamnese completa e anotação de todos os dados necessários. No Brasil, o Sistema de Informações de Agravos de Notificação (SINAN) tem por objetivo registrar e processar os dados sobre agravos de notificação em todo o território nacional, fornecendo informações para análise do perfil da morbidade e contribuindo, dessa forma, para a tomada de decisões nas esferas municipal, estadual e federal. O sistema é constituído por um conjunto de fichas padronizadas referentes à lista de doenças de notificação compulsória, entre elas a ficha de atendimento antirrábico humano. Nesta se classifica o acidente de acordo com as características do ferimento e do animal envolvido ${ }^{18}$.
Todos esses fatores devem ser analisados de forma muito criteriosa para que não sejam instituídos tratamentos desnecessários que, além de risco à saúde, podem gerar desperdícios aos cofres públicos. No Brasil, em 2005, o Ministério da Saúde gastou com as ações de vigilância epidemiológica para a raiva cerca de $\mathrm{R} \$ 66,4$ milhões, sendo grande parte desses recursos destinados à aquisição de imunobiológicos para profilaxia pós-exposição em humanos ${ }^{19}$.

De acordo com Chang et al..$^{20} \mathrm{e}$ Patrick \& O'rourke $^{21}$, os custos diretos e indiretos relacionados ao tratamento médico dos acidentados são elevados, consumindo recursos que poderiam ser aplicados em programas de promoção à saúde que atenderiam um grande número de pessoas.

\section{Metodologia}

\section{Caracterização do Município alvo do presente estudo}

O Município de Jaboticabal pode ser considerado como área de raiva controlada, uma vez que atende as prerrogativas estabelecidas para tal ${ }^{16}$. O programa de controle da doença contempla campanhas anuais de vacinação de cães e gatos, realizadas pela prefeitura; auxílio no controle populacional por meio de castrações realizadas por equipes da Associação Protetora dos Animais, da Faculdade de Ciências Agrárias e Veterinárias (UNESP/Jaboticabal), com apoio da prefeitura municipal; verificação de circulação viral, por meio de remessa ao Instituto Pasteur de tecido cerebral de cães e gatos mortos $(0,2 \%$ da população animal estimada, o que se traduz em média de 35 animais por ano); e atividades de educação em saúde.

O serviço de profilaxia da raiva humana concentra-se no CIAF VI (na época do levantamento para a presente pesquisa, no Centro de Saúde I "Albertino Affonso"), em dias úteis, e no Pronto Atendimento Municipal, nos finais de semana e feriados, onde são atendidas as pessoas que se envolvem em agravos com animais. O Pronto Aten- 
dimento presta os primeiros socorros e faz uma dose da vacina, quando necessário, encaminhando o paciente para a continuidade do tratamento no CIAF VI. Todos os acidentes notificados são registrados nas fichas de atendimento antirrábico humano, por atendentes de enfermagem, agentes de saúde ou pelo médico; a decisão da instituição do tratamento profilático é do médico responsável. Os dados são repassados para o SINAM e as fichas arquivadas com o prontuário do paciente.

No Município de Jaboticabal, como em todo o Estado de São Paulo, utiliza-se a vacina antirrábica humana preparada a partir de vírus cepa WISTAR, PM/WI38-1503-3M cultivado sobre células VERO e inativado com beta-propiolacto, fabricada pelo SANOFI PASTEUR S.A., importada, embalada e registrada pelo Instituto Butantan ${ }^{16,17}$.

\section{Estudo descritivo}

Para o presente estudo foi realizado um levantamento de dados registrados nas fichas de investigação de atendimento antirrábico humano do Município de Jaboticabal. A pesquisa foi autorizada pelo secretário de saúde, após solicitação oficial.

\section{Obtenção e digitalização dos dados}

As fichas de investigação de atendimento antirrábico humano estavam arquivadas na pasta (prontuário) de cada paciente. Para facilitar seu resgate, a Secretaria de Vigilância Epidemiológica do Município de Jaboticabal disponibilizou uma lista do Sistema de Informações de Agravos de Notificação (SINAN) contendo os nomes das pessoas envolvidas em acidentes com animais e que procuraram atendimento durante o período de estudo. A partir desta, efetuou-se uma pesquisa no computador do Centro de Saúde que gerou os números dos prontuários dessas pessoas. A ficha de investigação de atendimento antirrábico era extraída da pasta de cada um dos pacientes e, após imediata digitalização dos dados, devolvida ao arquivo.

\section{Formação do banco de dados}

Foram analisadas 3.056 fichas, relativas ao período de $1^{\circ}$ de janeiro de 2000 a 31 de dezembro de 2006.

A ficha é composta por 64 campos distribuídos em seis sessões diferentes. Para a presente investigação foram utilizados os seguintes campos: espécie do animal agressor; condição do animal no ato do acidente e durante o período de observação; conduta relacionada ao agravo: número de doses indicadas ao paciente e observação do animal, ou apenas observação do animal; interrupção do tratamento e motivo da interrupção; e indicação de soro antirrábico.

\section{Tabulação dos dados}

Os dados foram tabulados em planilhas do software Microsoft Office Excel 2003 ${ }^{22}$.

\section{Cálculo de custos}

Foram considerados apenas os custos com vacinas contra raiva humana destinadas à profilaxia pós-exposição. $\mathrm{O}$ custo da vacina contra raiva humana de cultivo celular, que foi a vacina utilizada durante o período estudado, manteve-se em torno de $\$ 10$ dólares a dose $\mathrm{e}^{23}$. Definidos os números de doses utilizadas a cada ano, multiplicou-se pelo valor médio anual do dólar comercial (venda) cotado em cada período ${ }^{24}$.

\section{Resultados e Discussão}

\section{Avaliação da conduta profilática adotada}

No período estudado, das 3.056 pessoas envolvidas em acidentes com animais, 2.493 foram submetidas à profilaxia com uso de vacina contra raiva. Somente 563 casos $(18,4 \%)$ foram conduzidos apenas com indicação de observação do animal, sem instituição de vacina. Pode-se comparar com o resultado (39\%) de Pinto \& Alleoni ${ }^{25}$, na mesma situação, índice ainda considerado baixo. Segundo a pesquisa realizada por Carvalho et al. ${ }^{26}$, a conduta adotada de apenas observação do animal agressor, sem 
vacinação, foi de $59,1 \%$ dos casos atendidos; Garcia et al. ${ }^{27}$ detectaram 50,6\%.

O número de indicações de profilaxia pós-exposição em Jaboticabal pode ser considerado muito alto $(81,6 \%$ das pessoas foram tratadas) quando comparado com resultados encontrados por Pinto \& Alleoni ${ }^{25}$ (59,86\%) em sub-regiões administrativas do Estado de São Paulo; Garcia et al. ${ }^{27}(41,9 \%)$ na grande São Paulo; Carvalho et al. ${ }^{26}(42 \%)$ em Maringá/PR; Rigo \& $\operatorname{Honer}^{29}$ (52,2\%) em Campo Grande/MS; e por outros autores estrangeiros ${ }^{28,30,31}$. No Estado do Paraná, no ano de 1999, 22.897 pessoas procuraram médico devido a algum tipo de agravo e, dessas, $54 \%$ receberam tratamento antirrábico pós-exposição ${ }^{32}$. No Brasil, a porcentagem de tratamentos a pessoas agredidas foi de $62,4 \%$ em 1999 e 56,06\% em $2001^{33}$. No Estado de São Paulo, a porcentagem de tratamentos, em 2007, foi de $35 \%^{37}$. Os registros disponíveis nos órgãos do Ministério da Saúde demonstram que, em média, 400 mil pessoas são atendidas, anualmente, no Brasil, vítimas de agravos por animais; no ano de 2009, foram 450 mil; mais de 300 mil (65\%) foram submetidas a tratamento antirrábico $^{34-36,52}$.

Em alguns trabalhos realizados no Estado do Ceará os resultados foram semelhantes aos de Jaboticabal, pois a porcentagem de pessoas encaminhadas para tratamento foi de $78,61 \%^{38}$ e $80,5 \%^{39}$. Entretanto, deve-se ressaltar a grande diferença da condição epidemiológica da raiva naquele Estado, quando comparada com a do Município de Jaboticabal; no Ceará, a raiva se mantém endêmica, tanto entre os cães como em animais silvestres. Pesquisas no exterior, como aquela realizada por Tomasiewicz et al ${ }^{40}$ na província de Lublin, na Polônia, apontam apenas $14,98 \%$ dos agredidos recebendo vacina contra raiva; de acordo com Gonzalez et al. ${ }^{41}$, apenas $3,17 \%$ dos agredidos receberam vacina contra raiva no Estado de Baja Califórnia, no México. Sadkowska-Todys et al. ${ }^{42}$ afirmaram que a Polônia é um país que tem muitos problemas com raiva, principalmente em animais silvestres, e que cerca de $0,02 \%$ da população total do país recebe vacina contra raiva em caso de agravo. Já em Jaboticabal, durante o período estudado, $0,5 \%$ da população recebeu vacina, mesmo que a doença não tenha sido detectada em nenhuma espécie animal desde o ano de 1992.

O tratamento pode ser interrompido, quando for o caso, mas, quando necessário, deve ser realizado até o final. Em Jaboticabal, entre os tratamentos interrompidos, apenas $4,1 \%$ foram por abandono do paciente, demonstrando que as pessoas seguem a profilaxia prescrita; esses resultados foram semelhantes aos encontrados por Pinto \& Alleoni ${ }^{25}$; Garcia et al..$^{27}$, Carvalho et al. ${ }^{26}$; Morais et al. ${ }^{38} \mathrm{e}$ Moreno et al..$^{39}$.

Com relação ao soro antirrábico, este foi indicado para 5,6\% dos agredidos, índice semelhante ao encontrado em Marília/ SP, Assis/SP e Tupã/SP, que foi de $3,4 \%^{25}$, porém maior que o encontrado em Lublin, na Polônia, que foi de $0,12 \%{ }^{40}$.

A conduta a ser seguida para a profilaxia da raiva humana, quando um indivíduo é exposto ao risco de contrair a raiva, está descrita nas Normas Técnicas (nacionais) para tratamento antirrábico humano ${ }^{18} \mathrm{e}$ no Manual Técnico do Instituto Pasteur ${ }^{16}$. Após análise criteriosa do acidente, pode-se chegar à correta instituição da profilaxia. De acordo com as normas do Instituto Pasteur, deve-se analisar a espécie envolvida, a condição clínica do animal no momento da agressão e a situação epidemiológica da área onde ocorreu o acidente. Estas informações são as mais importantes a serem consideradas para início de profilaxia adequada ${ }^{16,32}$, lembrando que a condição clínica é tomada como parâmetro apenas para cães e gatos, pois somente nestas espécies a evolução e a patogenia da doença são bem conhecidas.

Para analisar e questionar a conduta de profilaxia pós-exposição em humanos adotada em Jaboticabal no período de estudo, tomou-se o cuidado de levar em conta apenas os dados das fichas de atendimento antirrábico relativas a agravos causados por cães e gatos.

Considerando que o Município é área controlada e que 2.184 agravos foram causa- 
dos por cães e gatos com proprietário, e clinicamente sadios no momento do acidente e durante todo o período de observação, sugere-se que todos esses pacientes poderiam ter sido dispensados do tratamento com vacina; entretanto, apenas 464 casos foram encerrados e o restante, 1.720 pessoas, receberam pelo menos três doses de vacina. Os resultados e a análise indicam que a instituição de profilaxia pós-exposição, na maioria dos casos, foi feita com base apenas na caracterização do agravo, ou seja, local do ferimento, tipo e gravidade da lesão, e idade do agredido. De um modo geral, não foi considerada a condição epidemiológica da doença no município nem a condição do animal no momento da agressão.

A avaliação desses itens é muito importante, e isso foi demonstrado na pesquisa de Bentanzos Lopez et al. ${ }^{43}$ realizada em Chiapas, no México, quando analisaram 28 acidentes graves com mordedura de cães; como os agredidos residiam em áreas de raiva controlada e a estrita vigilância do animal era realizada, não foi implantado nenhum tipo de tratamento e ninguém desenvolveu a doença.

A indicação de profilaxia pós-exposição pode também ser equivocada, como descrito por Oliveira et al. ${ }^{44}$ em um estudo retrospectivo realizado no Estado de Minas
Gerais durante os anos de 1999 a 2004, onde se constatou a indicação excessiva de tratamentos pós-exposição em áreas de baixo e médio risco para a doença, e baixa indicação em áreas de alto risco.

A integração dos serviços médico e médico veterinário, a análise do tipo e das circunstâncias da exposição, a avaliação do animal potencialmente transmissor do vírus e a avaliação do risco epidemiológico da raiva na região de sua procedência, são fatores decisivos para adoção de conduta adequada $^{16}$.

\section{Análise do custo com vacinas}

Foram utilizadas 7.108 doses de vacina contra raiva humana em Jaboticabal no período de estudo, correspondendo a 2,85 doses por pessoa tratada (resultado semelhante ao encontrado por Morais et al. ${ }^{38}$ no Estado do Ceará).

O custo equivalente ao total de doses foi de R\$ 179.105,14 (Tabela 1).

Como já foi considerado no tópico anterior, 1.720 pessoas poderiam ter sido dispensadas da vacinação, com segurança, uma vez que no Município de Jaboticabal existe um serviço de vigilância epidemiológica eficaz, a cobertura vacinal nas campanhas de vacinação antirrábica urbana é

Tabela 1 - Custo total das doses de vacina contra raiva humana utilizadas nos anos de 2000 a 2006 no Município de Jaboticabal, SP, Brasil, de acordo com o valor médio do dólar comercial de venda em cada ano

Table 1 - Total cost of rabies vaccine shots administered in 2000 through 2006 in the Municipality of Jaboticabal, SP, Brazil, in accordance with the average value of the commercial dollar for sale in each year.

\begin{tabular}{lccc}
\hline Ano & $\begin{array}{c}\text { Número de doses } \\
\text { utilizadas }\end{array}$ & $\begin{array}{c}\text { Valor médio anual } \\
\text { do dólar }\end{array}$ & $\begin{array}{c}\text { Custo Total } \\
\text { (\$10 dólares/dose) }\end{array}$ \\
\hline 2000 & 1067 & $\mathrm{R} \$ 1,8302$ & $\mathrm{R} \$ 19.528,23$ \\
2001 & 1229 & $\mathrm{R} \$ 2,3504$ & $\mathrm{R} \$ 28.886,41$ \\
2002 & 1024 & $\mathrm{R} \$ 2,9212$ & $\mathrm{R} \$ 29.913,08$ \\
2003 & 1048 & $\mathrm{R} \$ 3,0783$ & $\mathrm{R} \$ 32.260,58$ \\
2004 & 869 & $\mathrm{R} \$ 2,9259$ & $\mathrm{R} \$ 25.426,07$ \\
2005 & 917 & $\mathrm{R} \$ 2,4352$ & $\mathrm{R} \$ 22.330,78$ \\
2006 & 954 & $\mathrm{R} \$ 2,1761$ & $\mathrm{R} \$ 20.759,99$ \\
\hline Total & 7108 & & $\mathrm{R} \$ 179.105,14$ \\
\hline
\end{tabular}


Tabela 2 - Custo das doses de vacina contra raiva humana possivelmente utilizadas de forma desnecessária nos anos de 2000 a 2006 no Município de Jaboticabal, SP, Brasil, de acordo com o valor médio do dólar comercial de venda em cada ano

Table 2 - Cost of rabies vaccine shots possibly misused in 2000 through 2006, in the Municipality of Jaboticabal, SP, Brazil, in accordance with the average value of the commercial dollar for sale in each year

\begin{tabular}{lccc}
\hline Ano & $\begin{array}{c}\text { Número de doses } \\
\text { possivelmente } \\
\text { desnecessárias }\end{array}$ & $\begin{array}{c}\text { Valor médio anual } \\
\text { do dólar }\end{array}$ & $\begin{array}{c}\text { Custo Total } \\
\text { (\$10 dólares/dose) }\end{array}$ \\
\hline 2000 & 788 & $\mathrm{R} \$ 1,8302$ & $\mathrm{R} \$ 14.421,97$ \\
2001 & 752 & $\mathrm{R} \$ 2,3504$ & $\mathrm{R} \$ 17.675,00$ \\
2002 & 650 & $\mathrm{R} \$ 2,9212$ & $\mathrm{R} \$ 18.987,80$ \\
2003 & 637 & $\mathrm{R} \$ 3,0783$ & $\mathrm{R} \$ 19.608,77$ \\
2004 & 502 & $\mathrm{R} \$ 2,9259$ & $\mathrm{R} \$ 14.688,01$ \\
2005 & 617 & $\mathrm{R} \$ 2,4352$ & $\mathrm{R} \$ 15.025,18$ \\
2006 & 644 & $\mathrm{R} \$ 2,1761$ & $\mathrm{R} \$ 14.014,08$ \\
\hline Total & 4590 & & $\mathrm{R} \$ 114.420,81$ \\
\hline
\end{tabular}

superior a $80 \%$ em cães, efetua-se a remessa de material para análise da circulação viral anualmente $(0,2 \%$ da população canina $\mathrm{e}$ felina), e não há registro de caso de raiva canina há mais de 15 anos, garantindo o status epidemiológico de área de raiva controlada.

As 4.590 doses de vacina utilizadas perfazem desnecessariamente um custeio adicional de $\mathrm{R} \$ 114.420,18$ (Tabela 2), montante que poderia ser investido em outros segmentos da Saúde Pública, sem prejuízo para a situação de controle da raiva no município.

Wada $^{52}$, no IV Seminário do Dia Mundial da Raiva, em 2010, ressaltou o custo com a assistência nos casos de agressões por animais, estimando $R \$ 21,63$ por dose de vacina em cultivo celular, e R \$ 4,45 e R \$ 15,28 por cada mililitro de soro heterólogo e de soro homólogo, respectivamente.

Além do custo com vacinas, devem ser computados outros custos diretos relativos aos cuidados médicos, contemplando medicamentos e outras vacinas, agulhas, seringas, entre outros itens, além dos honorários profissionais. $\mathrm{O}$ custo indireto inclui as perdas salariais, a perda da capacidade produtiva do indivíduo, e os custos não médicos, que são os gastos com alimentação, transporte e moradia temporária, quando for o caso ${ }^{45,46}$. Tais despesas não foram computadas neste estudo; portanto, estima-se que o custo do controle da raiva humana em Jaboticabal seja, seguramente, muito maior. Bernard Vallat, Director Geral da Organização Mundial de Saúde Animal (OIE), afirmou que o tratamento de uma pessoa com mordedura supõe um custo de vinte a cem vezes superior ao de vacinar um cão e que, com apenas $10 \%$ dos recursos financeiros utilizados em nível mundial para tratar pessoas acometidas por mordedura de cão, os Serviços Veterinários poderiam erradicar a raiva dos animais e quase eliminar os casos em humanos ${ }^{53}$.

A utilização de antibióticos é importante, pois geralmente as agressões, principalmente as mordeduras, evoluem para um quadro infeccioso. Estima-se que $4 \%$ a $25 \%$ dos ferimentos causados por mordeduras de cães podem infeccionar, sendo os ferimentos nas mãos os que apresentam maior risco ${ }^{47-50}$. O Clostridium tetani pode ser encontrado na boca dos cães, motivo pelo qual se indica a profilaxia do tétano em casos de agravos ${ }^{16,51}$. A utilização de antibióticos e da vacina contra o tétano aumenta ainda mais o custo dos atendimentos às agressões. Por isso, deve ser ressaltada a importância de um programa de educação 
e posse responsável visando diminuir o número de agravos.

A instituição de um tratamento adequado, a rapidez na observação do animal e no repasse de informações dão qualidade à vigilância epidemiológica em caso de acidentes com animais ${ }^{29}$. De acordo com Pinto \& Alleoni ${ }^{25}$, o que pode explicar o índice tão alto de tratamentos efetuados é a inexistência de condições para observação dos animais agressores, a postura dos agentes das Unidades de Saúde - que preferem indicar o tratamento, na maioria das vezes evitável, ao invés de fazer um estudo minucioso do caso - a falta de um instrumento adequado para registros de dados e repasse das informações, o que dificulta a tomada de decisões.

No Manual Técnico do Instituto Pasteur $^{16}$, a recomendação é que a observação do animal agressor por 10 dias deve, preferencialmente, ser supervisionada por médico veterinário, podendo ser realizada pelo responsável e/ou proprietário, no próprio domicílio do animal ou pelo serviço municipal de controle de zoonoses, por visita domiciliar ou isolamento em canil público. Uma visita inicial para observar o animal no dia do agravo é fundamental e daria subsídio para a conduta no atendimento aos casos de agressão a humanos, especialmente por cães e gatos.

\section{Conclusões}

Tendo por base os resultados obtidos, algumas recomendações tornam-se necessárias para melhorar o programa de atendimento a pessoas envolvidas em agravos com animais no Município de Jaboticabal.

- O número de tratamentos profiláticos pós-exposição instituídos em seres humanos no período do estudo foi muito elevado (81,6\% das pessoas agredidas), quando comparado às médias do Estado de São Paulo (35\%) e do Brasil (65\%).

- A análise dos dados demonstrou que a normatização de profilaxia antirrábica pós-exposição em humanos envolvidos em agravos com cães e gatos, em cujas espécies a patogenia está bem estabelecida, não foi seguida com critério, uma vez que não se considerava a condição do animal no momento da agressão, nem a condição de área de raiva controlada do Município de Jaboticabal.

- Como conseqüência, houve um custeio adicional de R\$114.420,18, montante este que poderia ser investido em outros segmentos da Saúde Pública.

\section{Recomendações}

- Capacitação permanente da equipe responsável pelo atendimento antirrábico humano, visando o correto preenchimento da ficha de notificação.

- Conscientização das equipes de saúde pública sobre a importância da identificação e observação do animal agressor. A observação pode ser feita tanto no domicílio quanto no canil municipal, preferencialmente sob a supervisão do médico veterinário.

- Fortalecimento da integração dos serviços médicos e veterinários no atendimento às pessoas envolvidas em agravos com animais, visando uma análise criteriosa do tipo e das circunstâncias do agravo, das condições do animal agressor e do risco epidemiológico da doença no município, para que a decisão de se instituir ou não profilaxia antirrábica pós-exposição seja tomada com critério e segurança.

Observação: Não existiu nenhum tipo de conflito de interesses na execução e apresentação deste trabalho. Houve concordância e autorização da Secretária de Saúde do Município de Jaboticabal, Dra. Sonia Mara Ferri, para a publicação dos resultados desta pesquisa. 


\section{Referências}

1. Global Alliance for Rabies Control (GARC). About rabies. 2011. Disponível em http://www.rabiescontrol.net/EN/ About-Rabies.html. [Acessado em 11 de abril de 2011]

2. Wada MY. Dez anos de raiva humana no Brasil. IV Seminário do Dia Mundial da Raiva - Instituto Pasteur/SP. Águas de Lindóia. 28 de setembro de 2010. Disponível em http://www.pasteur.saude.sp.gov.br/ diadaraiva/wrd.htm. [Acessado em 10 de abril de 2011]

3. WHO - World Health Organization. Facts sheets. n.99, 2010. Disponível em http:/ / who.int/mediacentre/ factsheets/fs099/en/ [Acessado em 11 de abril de 2011]

4. Acha PN, Szyfres B. Rabia. Zoonosis y Enfermedades Transmisibles Comunes al Hombre y a los Animales. 3ed. vol. 2. Washington: Organización Panamericana de la Salud 2003; 2; 351-83.

5. Kaplan C, Turner GS, Warrell DA. Rabies: The facts. New York: Oxford University Press; 1986.

6. World Health Organization. Facts sheets. n. 99, 2010. Disponível em http://who. int/ mediacentre/factsheets/ fs099/en/. [Acessado em 10 de abril de 2011]

7. CEARÁ (Estado). Secretaria da Saúde do Estado do Ceará. Nota técnica de 25/04/05. Disponível em http:// www.saude.ce.gov.br [Acessado em 20 de outubro de 2005]

8. Schneider MC, Almeida GA, Souza LM, Morares NB, Diaz RC. Controle da raiva no Brasil de 1980 a 1990. Rev Saúde Pública 1996; 30(2): 196-203.

9. Passos ADC, Silva AAMCC, Ferreira AHC, Silva JM, Monteiro ME, Santiago RC. Epizootia de raiva na área urbana de Ribeirão Preto, SP, Brasil. Cad Saúde Pública 1998; 14(4): 735-40.

10. De Mattos CA, De Mattos CC, Rupprecht CE. Rhabdoviruses. In: Knipe DM, Howley PM. Fields Virology. Philadelphia: Lippincott Williams \& Wilkins; 2001. p. 1245-78.

11. BRASIL. Ministério da Agricultura, Pecuária e Abastecimento. Programa Nacional de Controle da Raiva dos Herbívoros e Outras Encefalopatias: Revisão sobre raiva em herbívoros. Disponível em http:/ / www. agricultura.gov.br/ [Acessado em 15 de março de 2009]

12. Belloto AJ. Situação da Raiva no mundo e Perspectivas de Eliminação da Raiva Transmitida pelo Cão na América Latina. In: Anais do Seminário Internacional de Raiva; 2000 out 15-20, São Paulo (Br). São Paulo: Instituto Pasteur; 2000. p. 3.

13. World Health Organization. Expert Committee on Rabies. Eight Report. Geneva; 1992. (WHO - technical report series, 824).

14. Rupprecht CE, Stohr K, Meredith C. Rabies. In: Williams ES, Barker IK. Infectious disease of wild mammals. Iowa: Iowa State University Press; 2001. cap. 1, p. 3-36.
15. Rupprecht CE, Hanlon CA, Hemachudha T. Rabies re-examined. The Lancet Infectious Disease 2002; 2(6): 327-43. Disponível em http://www.thelancet. com/search/results?search_mode=cluster\&search_ cluster=thelancet\&search_text1=rabies+reexamined\&x= $12 \& \mathrm{y}=15$. [Acessado em 15 de fevereiro de 2007]

16. SÃO PAULO (Estado). Secretaria de Estado da Saúde. Instituto Pasteur. Profilaxia da raiva humana. 2 ed. São Paulo: Instituto Pasteur, 2000. n. 4, 33p. (Manuais, 4).

17. Associação Médica Brasileira. Conselho Federal de Medicina. Sociedade Brasileira de Pediatria. Projeto Diretrizes: Vacina Contra - Raiva Humana. Disponível em: http://www.projetodiretrizes.org. br/projeto_diretrizes/120.pdf. [Acessado em 21 de setembro de 2007]

18. BRASIL. Ministério da Saúde. Normas técnicas de tratamento profilático antirrábico humano. Disponível em http://www.saude.rj.gov.br/Acoes/ NORMA_TECNICA_DA_RAIVA_2002.pdf [Acessado em 10 de junho de 2006]

19. BRASIL. Ministério da Saúde. Brasil atento a Raiva Humana. Disponível em: http://portal.saude.gov.br/ portal/saude/visualizar_texto.cfm?idtxt=25213 [Acessado em 8 de março de 2009]

20. Chang Y, Mcmahon JE, Hennon DL, Laporte RE, Coben JH. Dog bite incidence in the city of Pittsburgh: a capture-recapture approach. Am J Public Health 1997; 87(10): 1703-5.

21. Patrick GR, O'rourke KM. Dog and cat bites: epidemiologic analyses suggest different prevention strategies. Public Health Reports 1998; 113(3): 252-7.

22. Microsoft Corporation. Microsoft Office 2003 editions: product guide. 2003. 89p. Disponível em http:// download.microsoft.com/download/0/f/1/0f1d5b1f53bc-47c3-bf6f-ac6d67cf9766/ Office2003Guide_ WP.doc. [Acessado em 25 de setembro de 2007]

23. Takaoka NY. (Diretora Técnica do Instituto Pasteur de São Paulo). Comunicação Pessoal; 2007.

24. BRASIL. Instituto de Pesquisa Econômica Aplicada. IPEA. Taxa de Câmbio. Disponível em http: / / www.ipeadata. gov.br/ipeaweb.dll/MenuCtrl?SessionID=2018377738 \&Mod=MACRO\&Lang=Portuguese [Acessado em $10 \mathrm{de}$ janeiro de 2008]

25. Pinto CL, Alleoni ES. Aspectos da vigilância epidemiológica da raiva em sub-regiões administrativas do Estado de São Paulo, Brasil, 1982-1983. Rev Saúde Pública 1986; 20(4): 288-92.

26. Carvalho WO, Soares DFPP, Franceschi VCS. Características do atendimento prestado pelo serviço de profilaxia da raiva humana na rede municipal de saúde de Maringá-Paraná, no ano de 1997. Informe Epidemiológico do SUS 2002; 11(1): 25-35. 
27. Garcia RCM, Vasconcellos AS, Sakamoto SM, Lopez AC. Análise de tratamento antirrábico humano pósexposição em região da Grande São Paulo, Brasil. Rev Saúde Pública 1999; 33(3): 295-301.

28. Ostrowska JD, Zajkowska J, Krupa W, Pancewicz S, Kondrusik M, Grygorczuk S, et al. Evaluation of the prophylactic anti-rabies vaccination program for adults carried out by the Center for Rabies Prevention in Bialystok, 1992-2001. Medycyna Pracy 2003; 54(5): 45356.

29. Rigo L, Honer MR. Análise da profilaxia da raiva humana em Campo Grande, Mato Grosso do Sul, Brasil, em 2002. Cad Saúde Pública 2005; 21(6): 1939-45.

30. Kilic B, Unal B, Semin S, Konakci SK. An important public health problem: rabies suspected bites and posexposure prophylaxis in a health district in Turkey. Int $\mathrm{J}$ Infect Dis 2006; 10(3): 248-54.

31. Poerner AL, Pereira MJS. Epidemiology of human rabies post-exposure prophylaxis and relationship between rabies control strategies in Centro Sul area of Rio de Janeiro, Brazil. In: Anais da XVII Reunião Internacional de Raiva nas Américas; 2006 outubro 15-20; Brasília (Br). Ministério da Saúde; 2006. p.170.

32. PARANÁ (Estado). Secretaria da Saúde do Estado do Paraná. Boletim Epidemiológico, ano III, n. 11, 2000. Disponível em http://www.saude.pr.gov.br/vigiepi/ boletim/primavera_2000/raiva_humana.htm. [Acessado em 10 de abril de 2011]

33. Araujo FA. Situação epidemiológica da raiva - panorama brasileiro. In: Simpósio Internacional Programa de Treinamento Controle de Zoonoses e as Interações Homem-Animal; 2001; Embu (Br). São Paulo: Arca Brasil; 2001. p. 29.

34. BRASIL. Ministério da Saúde. Fundação Nacional da Saúde. Normas técnicas para tratamento antirrábico humano. Brasília: Ministério da Saúde; 2002.

35. BRASIL. Ministério da Saúde. Fundação Nacional da Saúde. Relatório anual de atividades do programa de controle da raiva. Brasília: Ministério da Saúde; 2002a.

36. Organización Panamericana De La Salud. Eliminación de la rabia humana transmitida por perros en America Latina: análisis de la situación. Washington, DC: OPAS; 2004, 71p.

37. Reichmann MLAB. Impacto de medidas de prevenção de agravos produzidos por animais da espécie canina, em carteiros da empresa de correios e telégrafos do Estado de São Paulo, no período de 2000 a 2004 [tese de doutorado]. São Paulo: Faculdade de Medicina Veterinária e Zootecnia, Universidade de São Paulo; 2007.

38. Morais NB, Vasconcelos DC, Alencar LMS, Rolim BN, Moreno JO. The prophylaxis profile of the human rabies in the state of Ceará. In: Anais da XVII Reunião Internacional de Raiva nas Américas; 2006 outubro 15-20; Brasília (Br). Brasília: Ministério da Saúde; 2006. p.169.
39. Moreno JO, Morais NB, Vasconcelos DC. The profile of the prophylaxis of human rabies in the regional cell of health of Caucaia - CE. In: Anais da XVII Reunião Internacional de Raiva nas Américas; 2006 outubro 1520; Brasília (Br). Brasília: Ministério da Saúde; 2006. p. 172.

40. Tomasiewicz K, Fota-Markowska H, Krzowska-Firych J, Krawczuk G. Post-exposure anti-rabies prophylaxis in Lublin province (Eastern Poland) in 2004-2005. Ann Agric Environ Med 2006; 13(2): 337-40.

41. Gonzalez FV, Quinonez RO, Araiza JAC, Sanchez CAD, Rubio DL. Acciones de vigilancia epidemiologica que permiten al estado de Baja Califórnia (Mexico) mantenerse por 24 años sin casos de rabia urbana 20012006. In: Anais da XVII Reunião Internacional de Raiva nas Américas; 2006 outubro 15-20; Brasília (Br). Brasília: Ministério da Saúde; 2006. p. 184.

42. Sadkowska-Todys M, Rosinska M, Smreczak M, Czerwinski M, Zmudzinski JF. Rabies surveillance, trends in animal rabies and human post-exposure treatment in Poland, 1990-2004. Eurosurveillance 2005; 10(11): 226-8.

43. Bentanzos Lopez R, Dominguez Zarate H, Gomez Montes J, Yanes Gamboa LM. Manejo de pacientes com lesiones graves provocadas por perro em áreas com ausência de casos de rabia canina, em el estado de Chiapas México. In: Anais da XVII Reunião Internacional de Raiva nas Américas; 2006 outubro 15-20; Brasília (Br). Brasília: Ministério da Saúde; 2006. P .48.

44. Oliveira VMR, Miranda CFJ, Moreira EC, Pereira PLL, Silva JA. Evaluation of the human antirabic service, Minas Gerais, Brazil, 1999 to 2004. In: Anais da XVII Reunião Internacional de Raiva nas Américas; 2006 outubro 15-20; Brasília (Br). Brasília: Ministério da Saúde; 2006. p. 164.

45. Shiwiff S, Sterner MJR, Parikh S, Bellomy A, Slate D. Estimating the direct and indirect cost of rabies postexposure prophylaxis. In: Annals 14 International Conference; 2003; Philadelphia (USA). Philadelphia: Thomas Jefferson University; 2003. p. 75.

46. Secoli SR, Padilia KG, Litvoc J, Maeda ST. Farmacoeconomia: perspectiva emergente no processo de tomada de decisão. Ciência e Saúde Coletiva 2005; 10: 287-96.

47. Griego RD, Rosen T, Orengo IF, Wolf JE. Dog, cat, and human bites: A review. J Am Acad Derm 1995; 33(6): 1019-29.

48. Presutti RJ. Prevention and treatment of dog bites. Am Fam Physician 2001; 63(8): 1567-72.

49. Taplitz RA. Managing bites wounds. Currently recommended antibiotics for treatment and prophylaxis. Postgraduate Medicine 2004; 116(2) . Disponível em http://www. postgradmed. com/ issues/2004/08_04/taplitz.htm. [Acessado em 21 de junho de 2007] 
50. Stefanopoulos PK, Tarantzopoulos AD. Facial bite wouns: management update. International J Oral Maxillofac Surg 2005; 34(5): 464-72.

51. Elias FM, Schulz AF, Jorge WA. Tratamento dos ferimentos faciais causados por mordeduras de cão. Rev Méd HU-USP 1999; 9(1): 5-14.

52. SÃO PAULO (Estado). Secretaria de Estado da Saúde. Instituto Pasteur. Dez anos de Raiva Humana no Brasil. IV Seminário do Dia Mundial da Raiva; 2010, outubro, 28 - 30; Águas de Lindóia, SP. Disponível em http://www. pasteur.saude.sp.gov.br/diadaraiva/wrd.htm. [Acessado em 10 de abril de 2011]
53. OIE - World Organization for Animal Health. Eliminar la rabia en los perros como método de control óptimo para prevenir la propagación de la enfermedad. 2009, march, 13: Paris, France. Disponível em http://www.oie.int/es/ buscar/. [Acessado em 10 de abril de 2011]

Recebido em: 11/08/2010 Versão final apresentada em: 29/05/2011 Aprovado em: 26/09/2011 